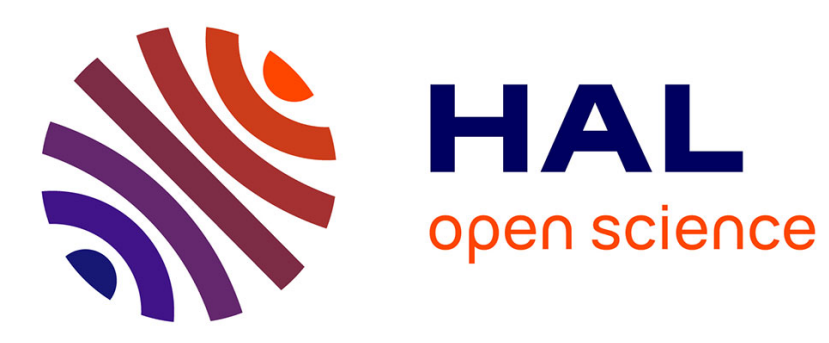

\title{
Le corps sans existence: les enfants victimes de néonaticides
}

Laurence Simmat-Durand, Natacha Vellut

\section{To cite this version:}

Laurence Simmat-Durand, Natacha Vellut. Le corps sans existence: les enfants victimes de néonaticides. CORPS : Revue Interdisciplinaire, 2013. hal-01984848

\section{HAL Id: hal-01984848 \\ https://hal.science/hal-01984848}

Submitted on 17 Jan 2019

HAL is a multi-disciplinary open access archive for the deposit and dissemination of scientific research documents, whether they are published or not. The documents may come from teaching and research institutions in France or abroad, or from public or private research centers.
L'archive ouverte pluridisciplinaire $\mathbf{H A L}$, est destinée au dépôt et à la diffusion de documents scientifiques de niveau recherche, publiés ou non, émanant des établissements d'enseignement et de recherche français ou étrangers, des laboratoires publics ou privés. 


\section{Le corps sans existence: les enfants victimes de néonaticides}

\section{Laurence Simmat-Durand}

\section{Natacha Vellut}

Cermes3, Université Paris Descartes- Ehess, CNRS UMR 8211, INSERM U988, Paris, France.

Le néonaticide est un crime particulier, le meurtre, en général par la mère, d'un nouveau-né dans ses premières heures d'existence. La procédure judiciaire débute généralement par la découverte du corps de l'enfant, ou par l'hospitalisation ${ }^{1}$ de la mère suivie de la recherche de ce cadavre dissimulé. Historiquement, c'est la première hypothèse qui est la plus courante, la seconde étant alors la clameur populaire ou la dénonciation à propos d'une femme que l'on avait vue enceinte (Tillier 2001). Comme le note Brouardel à propos des poursuites judiciaires de ce crime à la fin du XIXème siècle, " on trouve le cadavre d'un nouveau-né. Il a été étranglé, frappé d'un ou plusieurs coups de couteau. Le sentiment public est révolté, si en ce moment on trouvait la femme coupable d'un crime odieux, commis sur un innocent sans défense, alors que cet enfant est le sien, la femme elle-même serait en péril. Le cadavre est là, le juge, les témoins, l'expert ne voient que lui » (Brouardel 1897). Ce corps, que tous voient, a pourtant du mal à prouver son existence, comme nous allons le démontrer.

Le terme néonaticide, forgé par Resnick dans les années 1970, désigne cet infanticide particulier (Resnick 1970). Le mot désigne tout à la fois la mère et l'acte, mais aucun terme n'est créé pour désigner l'enfant qui en a été victime, qui n'est que cadavre. Depuis le nouveau code pénal de 1994, le meurtre d'un nouveau-né n'est plus une incrimination spécifique, il entre dans la catégorie des meurtres sur mineur de quinze ans (SimmatDurand, Vellut et al. 2012). Mais pour que cet enfant soit tué, il faut qu'il ait été reconnu comme vivant, car s'il est mort-né, "il n'est pas une personne au sens juridique du terme, les opérations prévues sur son corps ne sont pas soumis à réglementation. Ses funérailles sont possibles mais non obligatoires. Les familles ne sont pas tenues de les assurer » (Dumoulin and Valat 2011).

\footnotetext{
${ }^{1}$ Après son accouchement, la mère fait une hémorragie, ce qui entraîne son hospitalisation et la découverte d'un accouchement récent pour lequel elle ne peut pas " produire " l'enfant. Dans nos données, un tiers des affaires commence de cette façon, les dénonciations ne concernant que $6 \%$ des cas.
} 
Le présent article vise à essayer de définir le statut du corps de cet enfant, tué juste après qu'il ne vive et à montrer que ce corps est présenté de manière duelle, tout à la fois vivant et mort, ni vivant ni mort, selon l'existence que lui donne ou ne lui donne pas sa mère. II ne recevra véritablement d'existence légale que par l'intervention judiciaire qui le déclarera mort, ayant été vivant ou non, victime ou non de mort violente.

Les données sur lesquelles nous nous appuyons ici sont issues d'une recherche sur les morts violentes d'enfants de moins d'un an au travers de la presse française de 1995 à 2011. Environ huit cents décès suspects d'enfants ont été identifiés, qui ont donné lieu à plus de trois mille articles de presse. Parmi eux, 335 ont été tués à la naissance et dans 247 cas, la mère a été identifiée. Parmi ces affaires, plus d'une centaine sont documentées jusqu'au procès devant la cour d'Assises. Une analyse textuelle du contenu de l'information de la presse a été entreprise avec Nvivo9 nous permettant d'analyser ici tous les éléments livrés à propos du corps de l'enfant.

\section{La découverte du corps}

C'est le corps du bébé qui crée l'information, qui crée l'événement. Ce corps est mis en avant dans les titres des premiers articles couvrant l'affaire. "Le cadavre d'un bébé dans un tas de terre. " (Affaire Chatelaillon, 2001) ; "Bébé congelé dans les Côtes d'Armor " (Affaire Saint Nicolas du Pelem, 2007) ; "Découverte du cadavre d'un bébé » (Affaire Carentan, 2000) ; "Trois corps de nouveau-nés dans le jardin" (Affaire Contres, 2006). Le corps du bébé est alors au premier plan, il focalise l'intérêt des journalistes et de leurs lecteurs.

Le corps est le plus souvent découvert dans des lieux l'assimilant à un déchet : poubelle, toilettes, décharge, tas de gravats ${ }^{2}$ etc. " La macabre découverte d'un nouveau-né dans un sac poubelle " (Affaire Lucey 2009) apparaît comme une accroche "classique " des articles de presse relatifs aux néonaticides. Plus précis, plus sensationnel mais mettant en valeur et accentuant le même symbolisme de déchet, un article détaille : "elle a abandonné le cadavre de l'enfant, enfermé dans un sac plastique, dans un conteneur qui servait à jeter les déchets de la boucherie située au supermarché Lidl. Le corps avait été découvert le lendemain par un nomade qui fouillait dans les poubelles » (Affaire Melun, 1998).

Les symboliques du déchet, de la nourriture et de l'animalité peuvent être mêlées comme dans cette précision : "restes de bébé dans une cave près d'une assiette de mort aux rats " (Affaire Valognes, 2007). Autant de symboliques qui ne font pas du corps du bébé le corps d'un être humain.

Le lieu de découverte peut assimiler le corps du bébé à un corps mort alors même que l'enfant est encore vivant. Dans deux affaires, le corps d'un nouveau-né encore vivant est

\footnotetext{
${ }^{2}$ Sur 335 nouveau-nés découverts de 1995 à 2011, un quart l’a été à la poubelle.
} 
déposé dans un cimetière. Dans le premier cas, la petite fille ne peut être sauvée et décède (Affaire Le Plessis-Bouchard, 2010), dans le deuxième cas, la fillette survivra. Le lieu de découverte est interprété différemment en fonction de cette issue positive: "Pour le directeur du cimetière (...) le fait que le domaine soit à la fois excentré, à l'écart de toute habitation, mais aussi très passant tous les jours de l'année, peut être le signe d'un abandon "pour qu'on la trouve ". Assez calme pour permettre un dépôt furtif, assez fréquenté pour qu'un visiteur donne l'alerte " (Article du Parisien, 21 août 2004, hors corpus). Le geste est le même mais l'issue étant différente le lieu de découverte est ré-interprété, une nouvelle signification lui est accordé.

Des lieux peuvent représenter plusieurs symboliques contradictoires, comme ce bébé retrouvé dans un sac poubelle mais près d'une cathédrale (Affaire Beauvais, 2006).

La découverte peut avoir lieu dans des endroits insolites. "Le cadavre du nourrisson sera retrouvé quatre semaines plus tard à l'intérieur d'un classeur métallique rangé dans un débarras au domicile de la jeune femme » (Affaire Les Clayes sous bois, 1995).

La presse peut s'attarder sur l'effet ressenti par le découvreur du corps, surtout si celui-ci est extérieur à l'affaire : "J'ai senti une forte odeur comme de la charogne, témoigne cet homme bouleversé et encore sous le choc qui déclare ne plus s'alimenter et fumer déraisonnablement depuis sa terrible découverte. (...) J'ai alors vu le corps de l'enfant, avec les épaules qui sortaient du sac et les asticots qui commençaient à lui attaquer le visage " (Affaire Boe, 2007). Ici, le sensationnel, l'émotionnel, voire l'effet d'horreur et de fascination devant ce corps sont recherchés, tandis que d'autres traitements journalistiques accentuent le respect et la dignité qui seraient dus au corps humain. "Cet employé de la station-service Esso du Val de l'Eyre, située sur la N 10 à Belin-Beliet, a découvert le corps sans vie d'un bébé dans une cuvette des toilettes. Nu et abandonné. "Je travaillais. La journée a été longue et ne s'est pas passée comme une journée normale ", confie sobrement une salariée d'Esso encore émue. Une respectueuse discrétion a été observée par tous les employés autour de cette macabre découverte " (Affaire Belin Beliet, 2010).

La congélation ${ }^{3}$ ne préserve pas forcément le corps du bébé contrairement à ce que certains commentaires d'affaires de néonaticides pourraient laisser penser. Ainsi "c'est l'ancien concubin de la mère des enfants, venu chercher des meubles, qui a fait la macabre découverte, attiré par l'odeur pestilentielle qui se dégageait, les corps des bébés ayant manifestement subi à de nombreuses reprises des congélations et décongélations " (Affaire Albertville, 2006).

\footnotetext{
${ }^{3}$ Dans notre corpus, sur 335 affaires, seules 20 concernent des nouveau-nés placés au congélateur, ce qui en fait le lieu de découverte le plus rare, malgré la forte médiatisation de cet aspect depuis l'affaire Courjault.
} 
Le lieu de découverte du corps de l'enfant ne semble pas être en corrélation simple avec le mode opératoire du néonaticide. Dans l'affaire précédemment citée, relative à trois corps de nouveau-nés découverts dans un congélateur, il semble que le premier bébé soit décédé noyé dans la cuvette des WC où la mère venait d'accoucher, le second serait mort-né, et pour le troisième, la mère reconnaît des gestes actifs de meurtre, elle " a un vague souvenir d'avoir empoigné le corps et l'avoir empêché de crier ». (Affaire Albertville, 2006).

Le mode opératoire est essentiel à la Justice et permet la qualification pénale des affaires de meurtres. Mais le lieu et les conditions de découverte du corps de l'enfant victime de néonaticide permettent aussi une certaine catégorisation de celles-ci.

\section{Le corps ni vivant ni mort: l'objet}

Deux types de découvertes de néonaticides nous paraissent relever d'une appréhension du corps du bébé par la mère comme un « objet » ni vivant ni mort : la découverte dans des lieux l'assimilant à un déchet et la découverte dans un congélateur.

\section{Le corps du bébé comme objet-déchet}

Tout d'abord, avant d'être perçu comme un déchet, l'enfant n'est pas perçu comme vivant par la mère. "D'après elle [la mère], l'enfant ne bougeait pas " (Beauvais, 2006); "Après avoir accouché dans sa salle de bain, elle a mis son bébé sur elle, a coupé le cordon, sans se souvenir d'un cri quelconque. "Je croyais qu'elle était morte " (Affaire Chatelaillon, 2001).

Il est ensuite perçu comme un objet-déchet dont il convient de se débarrasser. "Elle a placé le petit corps dans un sac poubelle, entreposé dans la cour du domicile [... puis] jeté le sac poubelle dans la bouche d'égouts " (Affaire Chatelaillon, 2001). Le corps sera finalement retrouvé couvert de boue dans un tas de gravats.

"Quelque chose est sorti [...] pour moi ils n'étaient rien" (Affaire Valognes, 2007) le signifiant "bébé » ne s'impose pas, ne se présente pas. C'est le signifiant "rien " qui le remplace. C'est "quelque chose " qui apparaît aux yeux de la mère : un objet et non un sujet, un déchet et non un être. Comme les mort-nés de moins de six mois de grossesse, "Au regard de la loi, ils n'existent pas, leurs corps non plus. Juridiquement, ce sont des "riens", des "produits innommés", des "débris humains", des "choses" qui n'appartiennent à personne " (Dumoulin and Valat 2011). Ces débris anatomiques doivent aujourd'hui être incinérés.

" Elle sent quelque chose sortir de son ventre [...] Elle n'aurait pas eu conscience de mettre au monde un être humain ", le résume bien un article de presse. (Affaire Beauvais 2006)

Dans cette situation, l'enfant qui n'a pas été considéré comme un être humain, n'est pas 
considéré comme vivant, n'est pas envisagé comme ayant été tué : "Je n'ai pas tué mon bébé " explique la mère (Affaire Chatelaillon, 2001), effondrée, lors de son procès. Sa déclaration est à prendre au pied de la lettre : elle n'a pas tué son bébé car il n'était pas vivant et ce n'était pas un bébé, mais un objet et plus précisément un déchet.

"Elles n'accouchent pas d'un « bébé », pas davantage d'un fœtus, terme qui signifierait déjà que quelque chose de l'ordre de l'humain a pu s'inscrire ; mais elles éliminent de leur corps un rebut. » (Romano 2010)

Même si le vivant du bébé est subrepticement aperçu, entendu ou ressenti par la mère, il est rapidement étouffé, arrêté, stoppé. "J'ai eu juste eu le temps de baisser mon pantalon pour que ça arrive. Je n'ai pas regardé le sexe de l'enfant. Je mets une main derrière sa nuque et comme il se met à pleurer, je mets une main sur son visage pour que personne n'entende » (Affaire Orgon, 2008). Ce nouveau-né sera abandonné dans un sous-bois, déposé à même le sol, encore muni de son cordon ombilical. Il est l'objet qu'on laisse sur place, là où s'est déroulé l'accouchement, sans aucun geste attestant qu'il ait été considéré comme un être vivant.

\section{Le corps du bébé comme objet de la mère}

Dans les affaires de découverte du corps du bébé dans un congélateur, la mère a des difficultés à reconnaître l'enfant comme vivant.

"Sur les toilettes, elle expulse une masse importante [...] Rien ne bouge, elle va chercher un peignoir, revient, porte le nouveau-né dans la chambre de son fils. [...) A-t-elle étouffé l'enfant qu'elle venait de mettre au monde? Non martèle-t-elle depuis maintenant cinq ans [...] le scénario est toujours le même : après l'accouchement, ce jour de juillet 1995, le bébé n'a ni respiré, ni bougé, il n'a pas crié et son visage était étrangement teinté de bleu. Il était, assure l'accusée, mort-né " (Affaire Belin-Beliet, 1995). Cette version sera contredite par les experts légistes démontrant que l'enfant était né vivant et viable.

Cet enfant non vivant ne peut, comme le bébé-déchet précédemment décrit, avoir été tué. "Je n'ai jamais tué mon bébé " dit la mère (Affaire Belin-Beliet 1995). Le corps du bébé est placé dans un peignoir puis dans un sac poubelle puis dans le congélateur.

"Pour moi je n'ai pas donné naissance à un bébé, pour moi je n'étais pas enceinte » déclare la mère (Affaire Saint Nicolas du Pelem, 2007). Le corps du bébé est placé dans un sac poubelle noir puis au congélateur.

Ce corps d'enfant non vivant est envisagé par la mère comme un objet lui appartenant. En ce sens il n'est pas l'objet déchet dont il faut se débarrasser. II n'est pas un objet déchet qui 
se sépare du corps de la mère. Il est comme une partie de la mère, qu'elle préserve à ce titre.

«Cet enfant je le voulais ... je ne vis que pour les enfants... le congélateur c'était pour garder mon bébé près de moi » déclare la mère (Affaire Belin-Beliet, 1995).

La congélation du corps aurait pour fonction de figer le temps. Ces " objets-corps » figent le temps et créent l'illusion d'un temps arrêté, immobile. Ils renvoient à l'image des " corpsmorts " des marins qui permettent de garder le navire à un emplacement fixe " (Windels 2011). "C'est à moi [...] ils étaient en moi » dit la mère (Affaire Albertville, 2006) qui congèlera et recongèlera à plusieurs reprises les corps des nouveau-nés.

Le corps du bébé est un objet non séparé de la mère, malgré l'accouchement. " L'infanticide s'instituerait comme une tentative de récupération narcissique d'un objet auto-engendré et possédé jusque dans la mort » (Ravit 2011).

Dans ces deux premières "catégories ", nous n'avons pas affaire à des bébés mais à des objets, ni vivants ni morts. L'indistinction vivant/mort est en miroir avec l'indistinction présence/absence des corps dans le cas de conservation des corps dans des congélateurs. II n'y a pas « vraiment » de mort car il n'y a pas eu " vraiment " de vivant. A propos de deux cas de néonaticides où la mère a congelé ses nouveau-nés : « la vie n'est plus, et pourtant la mort n'est pas. II n'y a pas de mort possible, car la mort implique la putréfaction ou tout du moins la disparition du corps ; or, le corps reste toujours intact à la place où il a été déposé, c'est-à-dire dans le congélateur " (Windels, 2001). Notons cependant que la conservation par congélation ne garantit pas la conservation d'un corps intact. II y a aussi une indistinction des limites et de l'image du corps à conserver. Mais plus précisément, il nous semble que pour envisager le corps comme mort, il faut avoir pu l'envisager comme vivant.

Une autre hypothèse de Windels est que les mères qui congèlent leur bébé, les gardent pour se faire découvrir, en particulier des pères « ces femmes attendent une explication venue de l'extérieur? des paroles venant enfin leur dire ce qu'elles ont fait, ce qu'elles sont et ce qu'elles ont à faire? ». Wittgenstein a pensé l'opposition entre dire et montrer (Wittgenstein 1921 (1993 pour la présente édition)) et chez ces femmes à une immense difficulté de parole (Vellut 2012) répondrait une nécessité de montrer. II s'agirait de montrer pour dire sans les mots, sans une parole?

Ainsi, l'enfant est envisagé comme objet, soit qui se détache du corps de la mère en tant que déchet, soit qui appartient au corps de la mère et est à garder/conserver, il n'est pas un sujet. Dans la pratique clinique, on sait que si le parent se place comme prolongement du corps de son enfant, il l'empêche d'accéder au statut de sujet (Bergès 2010). 


\section{Le corps vivant puis mort : une ébauche de sujet}

Si le lieu de découverte du corps du bébé peut permettre de catégoriser différentes perceptions du corps de cet enfant, ce sont parfois non le lieu mais les conditions de cet abandon qui nous enseignent. Ainsi de cette "catégorie " que nous avons nommée des "bébés impossibles". Ce sont des bébés que les mères ne peuvent se permettre mais qu'elles perçoivent comme vivants et dont elles tentent de s'occuper dans les premiers instants de leur vie ou qu'elles n'abandonnent qu'avec difficulté.

\section{Le corps du bébé impossible, un corps vivant puis mort}

" J'ai pris ma petite sur moi [...] j'ai essayé de lui donner le sein ". Puis elle entreprend de la laver, dans une petite baignoire. "Elle s'est mise à hurler. C'était insupportable. Je n'arrivais pas à la calmer " Le bébé sera placé dans un sac poubelle puis dans une décharge. "Je ne voulais pas qu'elle meure ». (Affaire Carentan, 2000)

" J'ai eu peur qu'il [le bébé] souffre. Je ne voulais pas qu'il souffre. J'ai voulu le finir. [...] sa petite n'allait pas survivre " La mère tue son enfant, une petite fille, qu'elle prénommera Sonia. Elle pensait qu'un nouvel enfant allait contrarier son mari. "Lorsque sa fille aînée de 20 ans l'a découverte, la mère baignait dans son sang, victime d'une hémorragie. Immédiatement, la jeune fille a appelé les pompiers et à leur arrivée, la mère a été prise de panique et a tenté de s'enfuir, serrant le bébé inerte dans ses bras " Les parents donneront à l'enfant un prénom et une sépulture. (Affaire Saint Genis d'Hiesac, 2004)

"Je n'étais pas enceinte, je ne comprenais pas ce qui se passait. [...] je ne le touchais pas, il me faisait peur [...] Elle reste longtemps à côté du bébé qui n'a bougé selon elle "que quelques secondes. Je n'osais pas rentrer avec un bébé chez mes parents. Mais je ne voulais pas le laisser traîner dans la forêt et que les bêtes le mangent. "La nuit tombée, elle reprend sa route et en passant à Buswiller, voit " un escalier d'une maison qui donne sur la route. Je l'ai posé là " La mère sera poursuivie pour privation de soins ayant entraîné la mort et finalement acquittée par la cour d’Assises (Affaire Buswiller, 2005).

Ces situations peuvent être rapprochées de certaines affaires impliquant des mères souffrant de pathologies psychiatriques.

\section{Le corps du bébé et vivant et mort}

"La femme avait accouché chez elle dans la nuit de mercredi à jeudi. D’après ses 
déclarations, elle aurait aussitôt nettoyé l'enfant et lui aurait enfilé un pyjama. Elle aurait même cherché à l'allaiter. (...) Puis comme l'enfant s'est mis à pleurer, elle a pris un bain avec lui et a fin par le noyer volontairement " (Affaire Calais, 2006). Cette mère est diagnostiquée comme souffrant de troubles psychiques importants. Le corps du bébé est retrouvé à son domicile, sous un linge. Le corps n'est ni jeté, ni congelé, ni à proprement parler caché. Cette absence de stratégie vis-à-vis du corps de l'enfant signe l'impossibilité de l'envisager comme un « simple » objet.

Dans ces cas de « bébés impossibles " ou de bébés de mères au profil psychiatrique, le corps de l'enfant est envisagé comme le corps d'un être vivant. II est reconnu comme tel : " ma petite ", "l'enfant ", " un bébé ». Mais pour des raisons complexes, propres à l'histoire et à l'environnement de chacune de ces femmes, ce bébé ne peut s'inscrire dans une filiation et une dynamique de vie. Il est perçu comme vivant puis considéré comme mort, ou considéré en même temps comme vivant et mort dans un clivage spécifique à la psychose ou à une défense psychotique.

Dans une affaire relative à une mère très "désorganisée psychiquement ", "au passé dramatique ", il est noté que la mère avait envisagé un accouchement sous $X$, mais surprise par la naissance sur son lieu de travail, " elle a plongé dans l'horreur. Plaçant l'enfant dans un carton (...) l'amenant chez elle, l'enterrant vivant au fond du jardin. Trente centimètres de terre finissent par étouffer les cris. "Je les entends encore " murmure Martine " (Affaire Le Mans, 2001). Il y a bien tentative de considérer l'enfant comme vivant : elle entend ses cris, mais rapidement elle l'envisage comme mort et agit en conséquence par un enterrement.

\section{Le rôle de la procédure judiciaire : donner une existence à ce qui n'a pas eu d'existence}

Le rôle du procès pénal dans ces cas de néonaticides est de réinscrire le corps de l'enfant victime de meurtre en tant que mort, ce qui suppose qu'il ait vécu, ce qui introduit une temporalité dans sa brève existence. La mort qui n'était qu'apparente devient alors réelle, l'enfant bénéficie d'une existence légale et d'une inscription à l'Etat-civil (sauf s'il est déclaré mort-né). II réapparaît comme ayant été vivant, on lui donne un nom et un prénom et les rituels funéraires peuvent alors se dérouler. Selon l'attitude de la mère, elle y prendra ou non part, en choisissant le prénom, en demandant l'inscription sur le livret de famille. "Quand on les considère comme des mères criminelles, on les désigne comme des mères et l'on reconnaît l'existence de leur enfant qui ne peut plus être ravalé à n'être qu'un objet... " (Henry 2007). « Le jugement peut avoir un effet pacifiant » (Windels 2011). 
Quelquefois, la mère continue, malgré sa condamnation pour meurtre, à ne pas reconnaître l'existence de ce corps, le prénom et le nom sont choisis par les policiers ou les magistrats, l'enfant est enterré dans le carré des indigents ou les habitants se cotisent pour lui donner une sépulture. Cet enfant n'a alors définitivement pas d'existence dans le regard de sa mère.

Si on retient les distinctions suivantes pour définir le corps : corps comme soubassement de l'identité/corps comme éprouvé subjectif/corps comme expérience affective (Gernet 2007), seul le premier corps est " retrouvé ", " établi » lors du processus judiciaire. Le corps comme soubassement de l'identité. D'où l'importance d'attribuer un sexe à l'enfant, ce dont s'acquitte les médecins légistes, et de lui choisir un prénom, ce dont peuvent s'acquitter policiers ou magistrats si les parents n'accèdent pas à cette nomination.

Historiquement, dans notre culture, c'est le baptême qui faisait entrer l'enfant dans le monde des vivants : "Si malgré tous les efforts, le bébé n'a pu être baptisé avant de mourir, c'est le drame : il n'a pas reçu de nom et n'est intégré ni à la communauté des vivants, ni à celle des morts " (Morel, 2001). Ainsi, en Bretagne au XIXème siècle, lorsque la mère avait tué l'enfant à la naissance, elle affirmait l'avoir baptisé avant, afin d'éviter la mise au ban de la société (Tillier, 2001). Dans les affaires actuelles de néonaticides, le procès remplace le baptême dans cette fonction de faire entrer le corps de l'enfant dans la communauté des morts au titre d'un être humain qui a vécu.

\section{Conclusion}

Le corps comme " présence au monde » (Binswanger 1971) n'existe pas dans ces affaires. La notion de corps entraîne avec elle la notion de vivant, même si le corps mort conserve sa qualité de corps. Dans nos représentations immédiates, nous attendons d'un corps qu'il se meuve, vive, soit présent. Or, l'expérience corporelle qui «mobilise un sujet dans ses possibilités à se mouvoir et s'émouvoir " (Schmid Nichols 2010) n'a pu advenir. De plus, la notion de corps est attachée à la représentation d'un être humain, représentation qui est problématique dans ces affaires, le bébé étant plutôt envisagé comme un objet, un déchet, une partie du corps de la mère.

En ces sens, le corps du bébé victime de néonaticide n'a pas eu d'existence. II n'a pu être présence au monde, il n'est souvent même pas considéré comme " présent » par la mère qui l'envisage d'emblée comme mort-né. II ne peut accéder aux possibilités de se mouvoir et d'émouvoir, il n'accède pas à une expérience corporelle. 
Bergès, J. (2010). Les enfants hyperkinétiques Enfant insupportable. ERES: 39-56.

Binswanger, L. (1971). Introduction à l'analyse existentielle. Paris, Éditions de Minuit.

Brouardel, P. (1897). L'infanticide. Paris, Baillière.

Dumoulin, M. and A.-S. Valat (2011). "Morts en maternité : devenir des corps, deuil des familles." Etudes sur la mort 1(119): 77-99.

Gernet, I. (2007). Corps et subjectivité. L'Evolution Psychiatrique. France, Elsevier Masson SAS. 72: 338-345.

Henry, A. (2007). "Un témoignage clinique à propos des mères infanticides." Perspectives Psy 46(2): 135-140.

Ravit, M. (2011). "L'ombre de l'enfant mort Clinique de l'infanticide." Topique 117(4): 105115.

Resnick, P. J. (1970). "Murder of the newborn: A psychiatric review of neonaticide." American Journal of Psychiatry 126(10): 1414-1420.

Romano, H. (2010). "Meurtres de nouveau-nés et processus psychiques à l'œuvre chez les femmes néonaticides." Devenir 22( 4): 309-320.

Schmid Nichols, N. (2010). "Notre corps, cet étrange objet? Son importance dans le développement du sujet et dans le champ thérapeutique." Psychothérapies 30: 8995.

Simmat-Durand, L., N. Vellut, et al. (2012). "Les néonaticides devant la justice : le reflet d'une ambivalence face à ces crimes." Déviance et Société(4): in press.

Tillier, A. (2001). Des criminelles au village. Femmes infanticides en Bretagne (1825-1865). Rennes, Presses Universitaires de Rennes.

Vellut, N. (2012). "Néonaticides : des cris dans le silence de l'Autre." En-je lacanien(2): in press

Windels, M. (2011). "Mères sans alternative. Néonaticide et conservation par congélation." Cliniques méditerranéennes 84(2): 259-270.

Wittgenstein, L. (1921 (1993 pour la présente édition)). Tractatus Logico-philosophicus. Paris, Gallimard. 
Tableau des affaires citées avec leurs principales caractéristiques

\begin{tabular}{|c|c|c|c|c|c|c|c|c|c|}
\hline Lieu & Année & $\begin{array}{l}\text { Age } \\
\text { mère }\end{array}$ & $\begin{array}{l}\text { Enfants } \\
\text { tués }\end{array}$ & $\begin{array}{l}\text { Enfants } \\
\text { vivants }\end{array}$ & Lieu découverte & $\begin{array}{l}\text { Circonstances } \\
\text { découverte }\end{array}$ & Qualification procès & Décision judiciaire & $\begin{array}{l}\text { Détention } \\
\text { préventive }\end{array}$ \\
\hline Albertville & 2006 & 35 & 3 & 1 & Cave & Déménagement & Meurtre & 5 ans ferme & 8 mois \\
\hline Beauvais & 2006 & 21 & 1 & 0 & Poubelle & Aveux mère & Meurtre & $\begin{array}{l}\text { Acquittée puis en appel } 5 \\
\text { ans dont } 4 \text { avec sursis }\end{array}$ & 1 mois \\
\hline Belin-Béliet & 1995 & 35 & 1 & 3 & Congélateur & Dénonciation & Homicide volontaire & 10 ans ferme & 6 mois \\
\hline Belin-Béliet & 2010 & 19 & 1 & 0 & WC station essence & $\begin{array}{l}\text { Découverte } \\
\text { immédiate }\end{array}$ & En cours & En cours & Oui \\
\hline Boe & 2007 & ? & 1 & $?$ & Etang, fleuve & Découverte différée & & & \\
\hline Buswiller & 2005 & 20 & 1 & 1 & Lieu public & $\begin{array}{l}\text { Découverte } \\
\text { immédiate }\end{array}$ & Privation de soins & acquittement & \\
\hline Calais & 2006 & 36 & 1 & 0 & Salle de bains & $\begin{array}{l}\text { Tentative suicide } \\
\text { mère }\end{array}$ & & En cours & $\begin{array}{l}\text { Internée } \\
\text { d'office }\end{array}$ \\
\hline Carentan & 2000 & 21 & 1 & 1 & Poubelle & $\begin{array}{l}\text { Découverte } \\
\text { immédiate }\end{array}$ & Homicide volontaire & 8 ans ferme & 2 mois \\
\hline Chatelaillon & 2001 & 29 & 1 & 2 & Nature & Découverte différée & $\begin{array}{l}\text { Délaissement suivi } \\
\text { de mort }\end{array}$ & 5 ans dont 3 avec sursis & 8 mois \\
\hline Contres & 2000 & 39 & $\begin{array}{l}3 \text { (dont } \\
\text { jumeaux) }\end{array}$ & 4 & Jardin & Déménagement & Homicide volontaire & 5 ans avec sursis & Non \\
\hline Le Mans & 2001 & 35 & 1 & 2 & Jardin & Découverte différée & Homicide volontaire & 6 ans ferme & 1 mois \\
\hline
\end{tabular}




\begin{tabular}{|l|l|l|l|l|l|l|l|l|l|}
\hline Le Plessis & 2010 & 18 & 1 & 0 & Lieu public & $\begin{array}{l}\text { Découverte } \\
\text { immédiate }\end{array}$ & En cours & \\
\hline Les Clayes & 1995 & 30 & 2 & 2 & Débarras & Découverte différée & Meurtre & 12 ans ferme \\
\hline Lucey & 2009 & 35 & 1 & 0 & Etang & Découverte différée & En cours & \\
\hline Melun & 1998 & 25 & 1 & 1 & Poubelle & Découverte différée & $\begin{array}{l}\text { Coups et blessures } \\
\text { volontaires }\end{array}$ & 5 ans avec sursis \\
\hline Orgon & 2008 & 35 & 1 & 2 & Nature & Découverte différée & Meurtre & $\begin{array}{l}2 \text { ans ferme suivi socio- } \\
\text { judiciaire } 10 \text { ans }\end{array}$ \\
\hline St Genis & 2004 & 42 & 1 & 5 & Chambre & Hémorragie & Meurtre & 5 ans dont 4 ans et $4 \mathrm{~m}$ & 8 mois \\
\hline avec sursis
\end{tabular}

Source : base presse 1995-2011 